\title{
Cryptanalysis of an RFID Tag Search Protocol Preserving Privacy of Mobile Reader
}

\author{
Eun-Jun Yoon \\ Department of Cyber Security, Kyungil University, \\ 33 Buho-Ri, Hayang-Ub, Kyungsan-Si, Kyungsangpuk-Do 712-701, Republic of Korea \\ ejyoon@kiu.ac.kr
}

\begin{abstract}
RFID tag search system can be used to find a particular tag among numerous tags. In 2011, Chun et al. proposed an RFID tag search protocol preserving privacy of mobile reader holders. Chun et al. claimed that their proposed protocol can withstand five attacks to be considered in serverless search protocols, such as tracking, cloning, eavesdropping, physical, and Denial of Service (DoS) attacks. However, this paper points out that the Chun et al.'s protocol still can be vulnerable to the DoS attack.
\end{abstract}

Keywords: RFID, Privacy, DoS attack, Serverless search, Passive tag.

\section{Introduction}

Recently, Radio frequency identification (RFID) technology has been applied to many real-life applications [1]. Basically, RFID technology is used to identify RFID tags automatically. RFID tag search system can be used to find a particular tag among numerous tags [2,3]. RFID tag search system has many applications such as inventory management, supply chain, and search for books in the library. In 2009, Tan et al. [4] proposed secure serverless search protocols to treat the security and privacy concerns in RFID tag search system. Tan et al.'s protocols enable users with mobile readers to search specific tags even though the mobile readers cannot connect to a backend server. Tan et al.'s protocols also provide the robustness against the losses of mobile readers. Since mobile readers can be easily lost or stolen, the losses of mobile readers lead to leakage of sensitive information such as identifiers or secret keys of tags. Various RFID tag search protocols [5-9] have been proposed to meet security and privacy requirements based on Tan et al.'s protocols.

In 2011, Chun et al.[9] proposed a new RFID tag search protocol which can preserve privacy of mobile reader holders unlike related protocols. In the security analysis, Chun et al. claimed that their proposed protocol can withstand five attacks to be considered in serverless search protocols, such as tracking, cloning, eavesdropping,

\footnotetext{
Corresponding author.
} 
physical, and Denial of Service (DoS) attacks. However, this paper points out that the Chun et al.'s protocol still can be vulnerable to the DoS attack.

The paper is organized as follows. Section 2 reviews the Chun et al.'s RFID tag search protocol and then shows its weakness in Section 3. Finally, Section 4 concludes the paper.

\section{Review of Chun et al.'s RFID Tag Search Protocol}

The Chun et al.'s RFID tag search protocol is composed of two phases, which are an initial setup and a tag search. In the initial setup phase, from a backend server, each reader receives an access list of which each entry is encrypted with the identifiers of the reader and a tag. Then, in the tag search phase, the reader searches a specific tag using this list. Some of the notations used in the Chun et al.'s protocol are defined as follows:

- $R_{j}, T_{i}$ : Mobile RFID reader and tag, respectively.

- $R D_{j}, I D_{i}:$ Identity of $R_{j}$ and $T_{i}$, respectively.

- $S E=(E, D):$ Efficient symmetric encryption algorithm, e.g. AES-128.

$-t_{i}:$ Secret encryption key of the RFID tag $T_{i}$

$-\lambda$ : Bit length of a plaintext and a ciphertext.

$-x \leftarrow E_{t}(m):$ A deterministic polynomial-time algorithm that takes as input a symmetric key $t \in \kappa_{D}$ and a message $m \in\{0,1\}^{\lambda}$, outputs a ciphertext $x \in\{0,1\}^{\lambda}$.

- $m \leftarrow D_{t}(x):$ A deterministic polynomial-time algorithm that takes as input a private key $t$ and a ciphertext $x$, outputs a plaintext $m$.

- $\oplus$ : Bit-wise exclusive-OR (XOR) operation.

\subsection{Initial Setup Phase}

The phase consists of two parts. The first part is performed to generate information for an RFID tag $T_{i}$ and the second for a mobile reader $R_{j}$.

S.1 For each RFID tag $T_{i}$, the backend server generates a tag identifier $I D_{i}$ and a secret encryption key $t_{i}$ and then stores the pair $\left(I D_{i}, t_{i}\right)$ with the additional tag information into its own central database. Each tag $T_{i}$ stores the pair $\left(I D_{i}\right.$, $\left.t_{i}\right)$.

S.2 For a mobile reader $R_{j}$, the backend server generates an access list $L_{j}$ as follows: If the mobile reader $R_{j}$ is assumed to access to the tags $T_{i}(1 \leq i \leq n)$, the 
backend server computes each ciphertext $E_{t_{i}}\left(R D_{j} \oplus I D_{i}\right)$ for $i=1, \ldots, n$ by encrypting $R D_{j} \oplus I D_{i}$ with the secret key $t_{i}$ under the given encryption algorithm $E()$. Then, the backend server adds the pairs $\left(I D_{i}, E_{t_{i}}\left(R D_{j} \oplus I D_{i}\right)\right) \quad(1 \leq i \leq n)$ in the access list $L_{j}$. The backend server also transmits the access list $L_{j}$ to the mobile reader $R_{j}$ over a secure channel.

Table 1. Access list $L_{j}$ for a mobile reader $R_{j}$

\begin{tabular}{cc}
\hline$I D$ & $P W$ \\
\hline$I D_{1}$ & $E_{t_{i}}\left(R D_{j} \oplus I D_{1}\right)$ \\
$I D_{2}$ & $E_{t_{i}}\left(R D_{j} \oplus I D_{2}\right)$ \\
$\ldots$ & $\ldots$ \\
$I D_{n}$ & $E_{t_{i}}\left(R D_{j} \oplus I D_{n}\right)$ \\
\hline
\end{tabular}

\subsection{Tag Search Phase}

The Chun et al.'s tag search protocol is illustrated in Fig. 1 and is performed as follows:

$\mathrm{T} .1 \quad R_{j} \rightarrow T_{i}: \alpha \| n_{r}$

When $R_{j}$ wants to search $T_{i}, R_{j}$ first chooses a $\lambda$-bit random number $n_{r}$ and computes $\alpha=E_{I D_{i}}\left(R D_{j} \oplus n_{r}\right)$, then broadcasts $\alpha \| n_{r}$ to $T_{i}$.

T.2 $T_{i} \rightarrow R_{j}: \beta \| n_{t}$

Each tag $T_{i}$ who receives a message $\alpha \| n_{r}$ obtains $R D_{j}$ by computing $D_{I D_{i}}(\alpha) \oplus n_{r}=D_{I D_{i}}\left(E_{I D_{i}}\left(R D_{j} \oplus n_{r}\right)\right) \oplus n_{r}$ using its own identifier $I D_{i}$ and $n_{r}$. Then, each tag $T_{i}$ computes $K_{i}=E_{t_{i}}\left(R D_{j} \oplus I D_{i}\right) \oplus n_{r}$ with its own secret key $t_{i}$. Finally, each tag $T_{i}$ chooses a $\lambda$-bit random number $n_{t}$ and computes $\beta=E_{K_{i}}\left(I D_{i} \oplus n_{t}\right)$, then sends $\beta \| n_{t}$ to $R_{j}$.

T.3 $R_{j}$ computes $K_{i}=E_{t_{i}}\left(R D_{j} \oplus I D_{i}\right) \oplus n_{r}$ using the random number $n_{r}$ chosen before and the stored value $E_{t_{i}}\left(R D_{j} \oplus I D_{i}\right)$ in the access list $L_{j}$. Then, $R_{j}$ obtains $I D_{i}^{\prime}$ by computing $D_{K_{i}}(\beta) \oplus n_{t}=D_{K_{i}}\left(E_{K_{i}}\left(I D_{i} \oplus n_{t}\right)\right) \oplus n_{t}$ using $K_{i}$ and $n_{t}$. Finally, $R_{j}$ checks whether $I D_{i}^{\prime} ?=I D_{i}$ or not. If $I D_{i}^{\prime}==I D_{i}$ then $R_{j}$ knows that $T_{i}$ exists nearby $R_{j}$. 


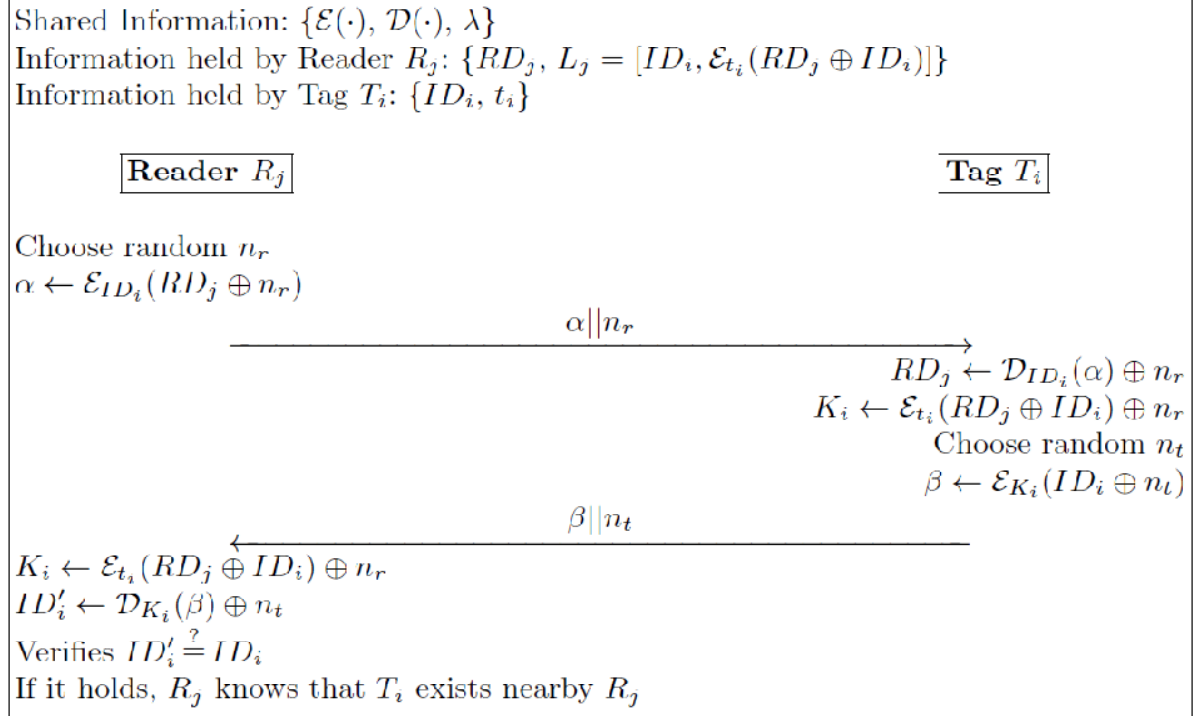

Fig. 1. Chun et al.'s RFID tag search protocol

\section{Denial of Service Attack against Chun et al.'s Protocol}

The Chun et al.'s RFID tag search protocol is vulnerable to Denial of Service (DoS) attack. DoS attack is one category of attacks on RFID systems. An adversary tries to find ways to fail target tag from receiving services. Almost all resources in an RFID system can become target of the DoS attack, including tag, reader, or backend server. Attacks on the air interface include shielding tags, flooding the reader field with a multitude of tags or selectively jamming the reader field. The goal is usually to sabotage specific resources of an RFID system, such a digital supply chain, effectively making the system unavailable to its intended users.

In the tag search phase, all tags $T_{i}$ nearby a mobile reader $R_{j}$ must respond to the request of $R_{j}$. Especially, in Step T.2 of the Chun et al.'s RFID tag search phase, all tags $T_{i}$ always must compute 3 times symmetric encryption operations to respond the request of $R_{j}$. These computations can be vulnerable to the following an adversary $A d v$ 's DoS attack which is illustrated in Fig. 2.

\section{A.1 $\operatorname{Adv} \rightarrow T_{i}: \alpha \| n_{r}$}

Suppose that an adversary $A d v$ intercepts $\alpha \| n_{r}$ from Step T.1. Adv broadcasts $\alpha \| n_{r}$ to $T_{i}$. 
A.2 $T_{i} \rightarrow A d v: \beta \| n_{t}$

All tags $T_{i}$ nearby $A d v$ will respond to the request of $A d v$ as follows:

Each tag $T_{i}$ who receives a message $\alpha \| n_{r}$ will obtain $R D_{j}$ by computing $D_{I D_{i}}(\alpha) \oplus n_{r}=D_{I D_{i}}\left(E_{I D_{i}}\left(R D_{j} \oplus n_{r}\right)\right) \oplus n_{r}$ using its own identifier $I D_{i}$ and $n_{r}$. Then, each tag $T_{i}$ will compute $K_{i}=E_{t_{i}}\left(R D_{j} \oplus I D_{i}\right) \oplus n_{r}$ with its own secret key $t_{i}$. Finally, each tag $T_{i}$ will choose a $\lambda$-bit random number $n_{t}$ and compute $\beta=E_{K_{i}}\left(I D_{i} \oplus n_{t}\right)$, then send $\beta \| n_{t}$ to $A d v$.

A.3 $A d v$ drops $\beta \| n_{t}$ and then broadcasts $\alpha \| n_{r}$ to $T_{i}$ continuously until success DoS attack.

Information held by $\Lambda$ dversary $\Lambda d v: \alpha \| n_{r}$
Information held by $\operatorname{Tag} T_{i}:\left\{I D_{i}, t_{i}\right\}$

Adversary $A d v$

Intercept $\alpha \| n_{r}$

\section{Tag $T_{i}$}

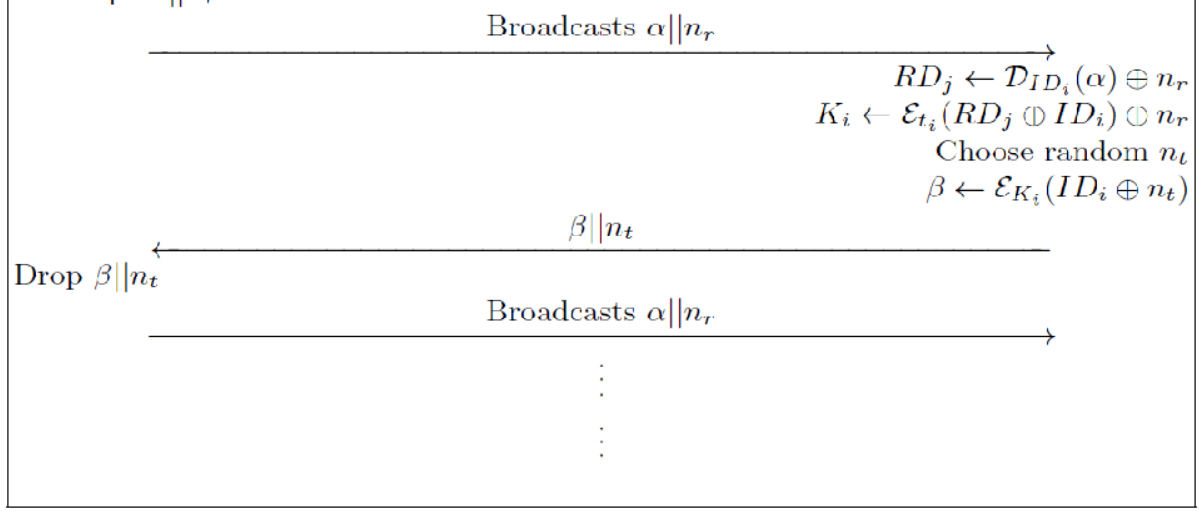

Fig. 2. DoS Attack against Chun et al.'s Protocol

From the computation results of above Step A.2, we can see that all tags $T_{i}$ automatically must compute 3 times symmetric encryption operations to respond the request of $A d v$. These 3 times symmetric encryption operations of the RFID passive tag $T_{i}$ can be quite expensive operations because $T_{i}$ uses a low-power microcontroller for sensing and communication with the RFID reader $R_{j}$.

Therefore, if $A d v$ broadcasts the intercepted $\alpha \| n_{r}$ continuously, all tags $T_{i}$ cannot respond to the request of the legitimate leaders. Moreover, $A d v$ can simply 
perform the above described DoS attack by choosing a random $\alpha^{*} \| n_{r}^{*}$ without intercepting the $R_{j}$ 's sending message $\alpha \| n_{r}$.

As a result, the Chun et al.'s RFID tag search protocol is vulnerable to the above described DoS attacks.

\section{Conclusions}

This paper analyzed the security of Chun et al.'s RFID tag search protocol preserving privacy of mobile reader holders. We presented a denial of service (DoS) attack against this protocol. Further works will be focused on improving the protocol which can not only withstand the DoS attack but also provide more computational efficiency.

Acknowledgments. We would like to thank the anonymous reviewers for their helpful comments in improving our manuscript. This research was supported by Basic Science Research Program through the National Research Foundation of Korea(NRF) funded by the Ministry of Education, Science and Technology(No. 2010-0010106). This study was also supported by the Intramural Research Support Program funded by the Kyungil University in 2012.

\section{References}

1. Radio Frequency Identification(RFID).: A focus on information security and privacy. OECDWorking Party on Information Security and Privacy, DSTI/ICCP/REG (2007)9/ FINAL, 70 (2008)

2. Vaudenay, S.: On Privacy Models for RFID. In: Kurosawa, K. (ed.) ASIACRYPT 2007. LNCS, vol. 4833, pp. 68-87. Springer, Heidelberg (2007)

3. Feldhofer, M., Wolkerstorfer, J.: Strong crypto for RFID tags-a comparison of low-power hardware implementations. In: Proc. 2007 IEEE International Symposium on Circuits and Systems (ISCAS), pp. 1839-1842 (2007)

4. Tan, C., Sheng, B., Li, Q.: Secure and serverless RFID authentication and search protocols. IEEE Trans. Wireless Commun. 7(4), 1400-1407 (2008)

5. Ahamed, S.I., Rahman, F., Hoque, E., Kawsar, F., Nakajima, T.: 3PR: secure server-less search protocols for RFID. In: Proc. 2nd International Conference on Information Security and Assurance (ISA), pp. 187-192 (2008)

6. Ahamed, S.I., Rahman, F., Hoque, E., Kawsar, F., Nakajima, T.: Secure and efficient tag searching in RFID systems using serverless search protocol. Int. J. Security and Its Applications 2(4), 57-66 (2008)

7. Won, T.Y., Chun, J.Y., Lee, D.H.: Strong authentication protocol for secure RFID tag search without help of central database. In: Proc. 2008 IEEE/IFIP International Conference on Embedded and Ubiquitous Computing (EUC), pp. 153-158 (2008)

8. Hoque, M.E., Rahman, F., Ahamed, S.I., Park, J.H.: Enhancing privacy and security of RFID system with serverless authentication and search protocols in pervasive environments. Wireless Personal Communications 55(1), 65-79 (2009)

9. Chun, L.J., Hwang, J.Y., Lee, D.H.: RFID tag search protocol preserving privacy of mobile reader holders. IEICE Electronics Express 8(2), 50-56 (2011) 